\section{Palliativmedizin/Palliative Care}

Cornelia Knipping (Hrsg.)

\section{Lehrbuch Palliative Care}

Bern: Hans Huber; 2006

740 Seiten, 62 Abbildungen, 46 Tabellen, kartoniert

Fr. 79.- / € 49.95

ISBN 978-3-456-84316-2

\section{Aulbert, Nauck, Radbruch (Hrsg.)}

\section{Lehrbuch der Palliativmedizin}

Stuttgart, New York: Schattauer; 2006

2., vollständig überarbeitete

und erweiterte Auflage

1448 Seiten, 225 Abbildungen, 208 Tabellen,

kartoniert

Fr. 195.- / € 129.-

ISBN 978-3-7945-2361-0

Die Palliative Care ist ein noch sehr junges Fachgebiet der Medizin und steht dennoch schon in politischer Brisanz. Die Palliativmedizin entwickelte sich seit der Eröffnung des ersten Hospizes 1967 in London durch Cicely Saunders rasant. Aus Modellprojekten wurden flächendeckende Netzwerke. Durch das gesteigerte gesundheitspolitische Interesse an Palliativmedizin und Hospizarbeit ist ein Wachstum und Ausbau des Konzepts zu erwarten.

Schon am Titel kann man den Hauptunterschied der beiden Werke erkennen. Das «Lehrbuch der Palliativmedizin» vom SchattauerVerlag wendet sich hauptsächlich an Ärzte, während das «Lehrbuch Palliative Care» vom Verlag Hans Huber in erster Linie für Pflegefachpersonen geschrieben wurde. Dennoch sollte dies keine all zu grosse Differenz ausmachen, da gerade im palliativen Bereich das Hauptaugenmerk auf der Interdisziplinarität liegt. Der aus dem Englischen stammende Begriff «care» meint auch nicht explizit Pflege, wie er hierzulande oft verstanden wird, sondern umfasst die medizinische Behandlung, Pflege und Begleitung von Patienten gleichermassen und sollte eher als ganzheitliches Konzept angesehen werden. Wenn das «Lehrbuch Palliativmedizin» auch inhaltlich den derzeit gültigen palliativmedizinischen Curricula für Ärzte entspricht, ist es aufgrund zahlreicher pflegerischer Hinweise auch für Krankenpflegekräfte geeignet und in der PalliativeCare-Ausbildung einsetzbar. Ebenso sollten Ärzte das gesamte Palliative-Care-Konzept, das erstmals umfassend, interdisziplinär, organisationsübergreifend sowie praxisorientiert im «Lehrbuch Palliative Care» dargestellt wurde, verinnerlichen, um in der Palliativmedizin ihr eigenes Profil weiterentwickeln zu können und eben nicht mehr vorrangig als Fachspezialisten zu fungieren.
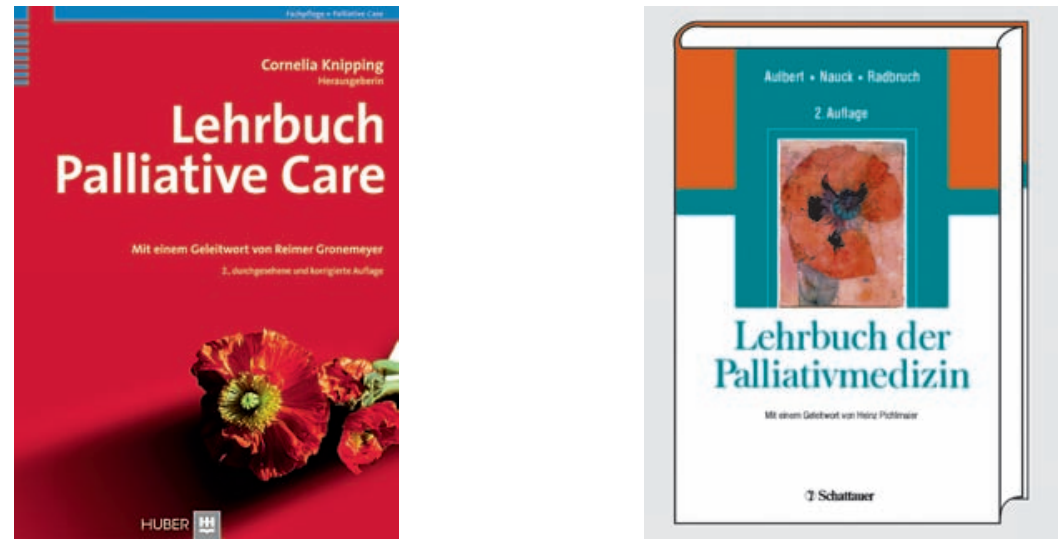

Beide Bücher haben auf der Titelseite die Mohnblume als Quelle des Morphins, das zum Symbol der Palliativmedizin geworden ist. Dass aber Palliativmedizin weit mehr ist als Schmerzlinderung durch Morphingabe, versteht sich nach der Lektüre der beiden Werke von selbst.

Im Autorenkollektiv des Schattauer-Lehrbuchs finden sich Ärzte, Therapeuten, Theologen, Psychologen, Sozialarbeiter und weitere Mitarbeiter aus Deutschland, der Schweiz, Österreich und den USA. Die Beiträge wurden von über 100 Mitwirkenden zusammengetragen. Im Autorenverzeichnis des Huber-Buches gibt es etwa halb so viele Autoren. Diese werden aber jeweils kurz mit ihrem Werdegang und ihrer Tätigkeit vorgestellt. Die Herausgeberin Cornelia Knipping und das internationale Autorenteam zeichnen sich durch langiährige Erfahrung und Expertise im Bereich der Palliative Care aus.

Das Palliative-Care-Lehrbuch regt durch seine Reflexionen und Ansätze zum Nachdenken an. Es fördert aktives Lernen sowie die Umsetzung und Anwendung der Lehrinhalte in der Praxis. Um den methodisch-didaktischen Ansatz zur selbständigen Auseinandersetzung ausschöpfen zu können, sollte man sich auf die Reflexionen und fachlichen Expositionen einlassen. Im Gegensatz dazu ist das Buch des Schattauer-Verlages eher ein Lehrbuch im konventionellen Sinne. Die Fakten werden didaktisch klar, gut strukturiert und übersichtlich dargeboten. Jedes Kapitel endet mit einer Zusammenfassung in Form von Kernaussagen. Im 12. Kapitel werden die Inhalte der Weiterbildungsordnung in der Palliativmedizin für Ärzte, Pflegepersonal und Fachkräfte aus psychosozialen Berufsgruppen vorgestellt.

Das «Lehrbuch der Palliativmedizin» erschien bereits 1997 in seiner Erstauflage und musste wegen der grossen Nachfrage 2000 nachgedruckt werden. Es handelt sich um das einzige umfassende Lehrbuch der Palliativmedizin, also explizit für Ärzte im deutschsprachigen Raum. Das gesamte Palliative-Care-Konzept mit besonderer Rücksicht auf eine würdevolle
Behandlung, Pflege und Begleitung wird erstmals umfassend im «Lehrbuch Palliative Care» dargestellt. Es versucht Haltung und Kultur bei der Behandlung und Versorgung unheilbar kranker, alter und sterbender Menschen beim Leser zu entwickeln. Die Betroffenen und ihre Familien stehen dabei im Mittelpunkt, ganz im Sinne des Zitates von Dörner: «Die Schwachen und Kranken zu achten und zu schützen ist die Würde der Gesunden.» Insofern stellt Palliative Care keine eigenständige Fachdisziplin dar, sondern sollte von allen Ärzten, Pflegenden und Personen in anderen Gesundheitsberufen als Konzept bei der täglichen Arbeit umgesetzt werden. Um würdevoll behandelt zu werden, sollte es nicht erst sterbenskranke Patienten brauchen. Alle Menschen und insbesondere die Kranken haben ein Recht auf respektvolle, ganzheitliche und individuelle Betreuung. In seiner Neuauflage versucht das «Lehrbuch der Palliativmedizin» dem Rechnung zu tragen. Das Patientenspektrum wird erweitert. Es werden zusätzliche Krankheitsgruppen in die palliativmedizinische Versorgung mit einbezogen.

Obwohl das «Lehrbuch der Palliativmedizin» seitenzahlmässig fast doppelt so umfangreich ist wie das «Lehrbuch Palliative Care», erscheint es durch das kleinere Format viel handlicher. Das Layout ist klar und übersichtlich gestaltet, zeichnet sich jedoch nicht durch etwas Besonderes aus. Wer es ansprechender mag, die kleinen Extras liebt, auf künstlerische Gestaltung und hohe Papierqualität Wert legt, wird das Buch des HuberVerlags zu schätzen wissen.

Beide Bücher sind durchaus lesens- und empfehlenswert für alle, die sich umfassend mit dem Thema Palliativmedizin auseinandersetzen und tiefgründig in diesen Bereich einblicken wollen. Die Qual der Wahl liegt also bei Ihnen.

S. Krüger, Lausanne 